\title{
Multiple sclerosis: disease modifying therapy and the human leukocyte antigen
}

Esclerose múltipla: terapêutica modificadora da doença e antígenos leucocitários humanos

\author{
Lineu Cesar Werneck¹, Paulo José Lorenzoni, Cláudia Suemi Kamoi Kay', Rosana Herminia Scola
}

\begin{abstract}
Objective: To investigate the potential relationship between the human leukocyte antigen (HLA) type (class I and II) and the response to several disease-modifying therapies (DMTs) in patients with multiple sclerosis (MS). Methods: We analyzed clinical data of 87 patients with MS at the beginning and end of each type of DMT including the disease duration, Expanded Disability Status Scale and Multiple Sclerosis Severity Score (MSSS). Genotyping of HLA-DRB1, HLA-DPB1, HLA-DQB1, HLA-A, HLA-B and HLA-C alleles were identified using high-resolution techniques. Statistical correlation between the HLA type and response to DMTs was done using the initial and final MSSS. Results: Statistical relationships ( $p$ 0.05) were found for only 15 of 245 alleles tested. There was a reduction in the MSSS for patients treated with corticosteroids (DRB1*15:01, DPB1*04:01, DQB1*02:01 and DQB1*03:01), azathioprine (DRB1*03:01, DPB1*04:01, DQB1*03:02, DQB1*06:02, HLA-C*07:02), interferon $\beta$-1a 22 mcg (DRB1*11:04, DQB1*03:01 and DQB1*03:02), interferon $\beta-1 \mathrm{a} 30 \mathrm{mcg}$ (DPB1*02:01, $H L A-C * 05: 01)$ and interferon $\beta-1 b(D Q B 1 * 02: 01)$. Conclusion: These findings suggest a few relationships between the HLA and response to DMTs in the disability for some types of HLA class I and II alleles in a specific subset of MS patients.
\end{abstract}

Keywords: Multiple sclerosis; biomarkers; pharmacogenetics; therapeutics.

\section{RESUMO}

Objetivo: Investigação da possivel relação entre os tipos dos antígenos leucocitários humanos (HLA) classes I e II e a reposta a diversas terapêuticas modificadores da doença na incapacidade (DMT) da esclerose múltipla (MS). Métodos: Foram estudados os dados clínicos de 87 pacientes com MS no início e no final de cada de cada DMT, incluindo o tempo de doença, EDSS e MSSS. Através de técnicas de genotipagem de alta resolução, foram identificados os alelos dos HLA-DRB1, HLA-DPB1, HLA-DQB1, HLA-A, HLA-B e HLA-C. Foram realizados estudos estatísticos entre os tipos de HLA e a resposta às DMT, utilizando os valores iniciais e finais do MSSS. Resultados: Foram encontradas relações estatísticas $(p<0.05)$ para somente 15 alelos de 245 analisados. Houve redução dos valores do MSSS em pacientes tratados com corticosteroides (DRB1*15:01, DPB1*04:01, DQB1*02:01 e 03:01), azatioprina (DRB1*03:01, DPB1*04:01, DQB1*06:02, DQB1*03:02, HLA-C*07:02), interferon $\beta-1$ a 22 mcg (DRB1*11:04, DQB1*03:01 e 03:02), interferon $\beta-1$ a 30 mcg (DPB1*02:01, HLA-C*05:01) e interferon $\beta-1 b$ (DQB1*02:01). Conclusão: Os dados sugerem poucas relações entre os alguns tipos de HLA classe I e II com a resposta às DMT na incapacidade em grupos específicos de pacientes com MS.

Palavras-chave: Esclerose múltipla; biomarcadores; farmacogenética; terapêutica.

Treatment of multiple sclerosis (MS) has progressed significantly in recent years and now includes immunosuppressants, immunomodulators, biologics and, more recently, medications that interfere with the release or maturation of lymphocytes. These medications have been shown to reduce the number of relapses, but the results in terms of reduced disability have been modest as most of the studies carried out were generally restricted to two years ${ }^{1,2}$.

Since the introduction of immunomodulators to treat MS, the response has been found to vary from patient to patient and with the research center where the study was conducted ${ }^{3}$. To find a factor that influences the evolution of MS in patients treated with disease modifying therapies (DMTs), several

${ }^{1}$ Universidade Federal do Paraná, Hospital de Clínicas, Serviço de Neurologia, Curitiba PR, Brasil.

Correspondence: Lineu Cesar Werneck; Hospital de Clínicas, Universidade Federal do Paraná; Rua General Carneiro, 181; 80060-900 Curitiba PR, Brasil. e-mail: lineu.werneck@hc.ufpr.br

Conflict of interest: Lineu Cesar Werneck: Conference fees from Merck-Serono, Bayer-Schering, Abbott-Biogen, Roche, TRB Pharma and BoehringerIngelheim; Travel grants to attend meetings from Bayer-Schering, Merck-Serono, TEVA, Abbott-Biogen, TRB Pharma, Genzyme; Fees for clinical trials from TRB-Pharma, Bayer-Schering, Abbott; Research grants from the Conselho Nacional de Pesquisas (CNPq), Fundação Araucária, NIH (Bethesda), Merck-Serono, Biogen; Productivity grants from CNPq 1A (PQ1A), Universidade Federal do Paraná (Senior Professor).

Paulo José Lorenzoni: There is no conflict of interest to declare. Cláudia Suemi Kamoi Kay: Travel grants to attend meetings from Novartis. Rosana Herminia Scola:Travel grants to attend meetings from Bayer-Schering, Merck-Serono, TEVA, Abbott-Biogen, Novartis and Genzyme; Conference fees from MerckSerono, Novartis and Genzyme.

Support: This study was supported by UFPR, CNPq, Serono and Biogen.

Received 15 February 2018; Received in final form 02 July 2018; Accepted 10 July 2018. 
attempts have been made to identify a relationship with the patient's immunogenetic profile, e.g. the human leukocyte antigen (HLA) type, but without success ${ }^{4,5,6,7,8}$. The HLA are glycoproteins present on the surface of lymphocytes of the major histocompatibility complex (MHC) and are responsible for the initiation of the immunological response for an antigen. The MHC expresses three different antigen-present cell classes of HLA. The MHC class I (HLA A, B and C) molecules present the peptides from pathogens, such as a virus, to CD8 cytotoxic cells. The CD4 T cells recognize the MHC class II (HLA DRB1, DPB1 and DQB1) in other cells of the immune system stimulating the B cells to produce antibodies ${ }^{9}$.

The genes that codify these HLA cell proteins are highly polymorphic and variable, according to the population studied, with some specific types related to $\mathrm{MS}^{9,10}$. The failure to find a relationship between the HLA type and response to DMTs may be due to the variation in the immunogenetic profiles of the populations studied and the different techniques used in the HLA typing has previously been reported ${ }^{10}$. However, some studies have shown an association between the presence of neutralizing antibodies and HLA alleles such as HLA-DRB1*03:01, HLA-DRB1*04:04, HLA-DRB1*11:0411 and the HLA-DRB1*07:01-DQB1*02:02 haplotype ${ }^{12}$. One study found a relationship between a better response to interferon $\beta$ and an increased frequency of HLA-DRB $1 * 04$, as well as between the HLA-A*03-B*44-DRB1*04 haplotype and decreased frequency of HLA-B* $15^{13}$. Another study found an association between the response to glatiramer acetate and HLA-DQB2, as well as other genes ${ }^{14}$.

To date, the relationship between the response to DMTs and the patient's HLA type, disease duration, age, disability and duration of DMT has not been well established. For this reason, in this study we investigated the associations between the HLA (class I and II) profile and disability progression (therapeutic response) in a group of MS patients treated with DMTs in a real-world outpatient clinic.

\section{METHODS}

We analyzed 87 patients ( 57 females and 30 males, 84 whites and three non-whites) with a diagnosis of relapsing-remitting MS based on the 2010 and 2013 revisions to the McDonald criteria $^{15,16}$. The patients were treated at the Demyelinating Disease Outpatient Clinic of the Neurology Service at the Hospital de Clínicas, Federal University of Paraná, Curitiba, Brazil. The MS patients who fulfilled the following criteria were included: 1) patient who had complete medical records available with information on the DMT used and disease duration at the beginning and end of each type of treatment; 2) patients must have had at least six months of therapy with only one DMT; 3) patients with more than one DMT at same time were excluded, excepted for the use of corticosteroid during exacerbation, but in these instances, they were placed only in the DMTs already in use, not in the corticosteroid group, and 4) patients with an available assessment on the Expanded Disability Status Scale (EDSS) ${ }^{17}$ or sufficient data in the patient's records to allow their disability to be graded at the beginning and end of each treatment. The MS patients who had incomplete medical records or conflicting data, or who had not been treated with DMTs were excluded (Table 1).

Data was recorded at the beginning and end of each type of treatment, including the disease duration and EDSS score, which allowed calculation of the Multiple Sclerosis Severity Score (MSSS) ${ }^{18}$ and correlation with the DMT used.

The MSSS score was obtained by the correlation of the degree of disability (EDSS) and duration of disease ${ }^{18}$. The patients with less than one year, were placed in the MSSS as year one and the score was correlated with treatment duration and the HLA genotype. The change in the MSSS between the beginning and end of each type of treatment (MSSS initial and MSSS final) was compared using the paired-sample Student's t-test $(\mathrm{t})^{19}$ or Wilcoxon signed-ranks test $(\mathrm{T})^{20}$, with SPSS-19 statistical software.

We chose a high-resolution technique for DNA analysis of the HLA system, because the old technique with low resolution shows only the serological result of the protein like the alleles group, but not the specific HLA protein ${ }^{21,22}$.

Briefly, as we described previously ${ }^{10}$, the genomic DNA was extracted from blood previously stored at $-70^{\circ} \mathrm{C}$, using a standard phenol-chloroform technique after treatment with proteinase-K and then amplified by polymerase chain reaction (PCR) to obtain gene fragments (exons) related to HLA class I and II using oligonucleotides flanking specific regions of the following exons: HLA-A (exons 2, 3 and 4), HLA-B (exons 2, 3 and 4), HLA-C (exons 2, 3 and 4), HLA-DRB1 (exon 2), HLA-DPB1 (exon 2) and HLA-DQB1 (exons 2 and 3). The PCR was performed separately for each exon of these different genes using a conventional method with Taq DNA polymerase (Abbott Molecular Diagnostics) and a sequence based typing (SBT) kit (AlleleSEQR-SBT) (Atria Genetics) following the manufacturers' instructions. The PCR products were purified by ExoSAP-IT" (USB, Cleveland, Ohio). The purified PCR products were then subjected to a second round of PCR using Big Dye Mix (Applied Biosystems), followed by purification of the products by the isopropanol method. The amplified fragments were directly sequenced in forward and reverse directions by fluorescent capillary electrophoresis using POP-6 polymer (Applied Biosystems, Foster City, CA) in an ABI PRISM 3100 and 3130 Avant Genetic Analyzers (Hitachi High-Technologies Corporation, Tokyo, Japan). The HLA sequences were compared with reference sequences by high-resolution HLA typing using Assign SBT software (Conexio-Genomics, Fremantle, Australia) and uTYPE* HLA Analysis Software (Thermo Fisher Scientific, Waltham, MA) ${ }^{10}$.

Of the 87 patients studied, 79 were tested for HLA-DRB1, 79 for HLA-DPB1, 77 for HLA-DQB1, 54 for HLA-A, 58 for HLA-B and 78 for HLA-C. Since each patient had two alleles, 
Table 1. Disease-modifying therapy and HLA. Gender, age, duration of symptoms, EDSS, MSSS and HLA alleles.

\begin{tabular}{|c|c|c|c|c|c|c|}
\hline HLA & DRB1* & DPB1* & DQB1* & $A^{*}$ & $B^{*}$ & $C^{*}$ \\
\hline $\mathrm{N}^{\circ}$ alleles tested & 50 & 28 & 36 & 38 & 55 & 36 \\
\hline Female (No. of patients) & 53 & 50 & 49 & 35 & 36 & 49 \\
\hline Male (No. of patients) & 26 & 29 & 28 & 19 & 22 & 29 \\
\hline White & 76 & 76 & 74 & 52 & 55 & 75 \\
\hline Non-white & 3 & 3 & 3 & 2 & 3 & 3 \\
\hline \multicolumn{7}{|l|}{ Age at treatment (years) } \\
\hline Mean & $33.88 \pm 10.39$ & $34.86 \pm 10.57$ & $34.30 \pm 10.66$ & $35.30 \pm 10.50$ & $36.26 \pm 10.48$ & $34.33 \pm 10.59$ \\
\hline Median & $33.00(12-60)$ & $35.00(12-61)$ & $34.00(12-63)$ & $36.00(12-60)$ & $35.00(12-63)$ & $34.00(12-63)$ \\
\hline \multicolumn{7}{|c|}{ Age at onset of symptoms (years) } \\
\hline Mean & $29.00 \pm 9.59$ & $29.95 \pm 9.99$ & $29.62 \pm 10.00$ & $30.56 \pm 9.81$ & $29.67 \pm 9.99$ & $29.86 \pm 10.04$ \\
\hline Median & $29.00(12-50)$ & $30.00(12-61)$ & $29.00(12-61)$ & $30.50(12-50)$ & $29.50(12-61)$ & $30.00(12-61)$ \\
\hline \multicolumn{7}{|c|}{ Duration of symptoms (months) } \\
\hline Mean & $64.44 \pm 71.13$ & $60.09 \pm 71.23$ & $61.75 \pm 71.64$ & $65.14 \pm 77.61$ & $67.53 \pm 78.90$ & $62.27 \pm 71.52$ \\
\hline Median & $48.00(0-373)$ & $36.00(0-373)$ & $39.00(0-373)$ & $39.00(0-373)$ & $47.00(0-373)$ & $40.50(0-373)$ \\
\hline \multicolumn{7}{|c|}{ Duration of treatment (months) } \\
\hline Mean & $46.17 \pm 46.13$ & $46.25 \pm 46.69$ & $45.63 \pm 46.55$ & $46.25 \pm 47.78$ & $49.51 \pm 51.12$ & $46.85 \pm 47.31$ \\
\hline Median & $30.00(6-276)$ & $30.00(6-276)$ & $28.00(6-276)$ & $29.00(6-276)$ & $\begin{array}{c}30.00 \\
(96-276)\end{array}$ & $29.50(6-276)$ \\
\hline \multicolumn{7}{|l|}{ EDSS (mean) } \\
\hline Initial & $2.62 \pm 1.92$ & $2.60 \pm 1.90$ & $2.60 \pm 1.83$ & $2.48 \pm 1.72$ & $2.46 \pm 1.87$ & $2.58 \pm 1.83$ \\
\hline Final & $3.07 \pm 2.26$ & $3.00 \pm 2.25$ & $2.96 \pm 2.15$ & $2.85 \pm 2.06$ & $2.88 \pm 2.20$ & $3.00 \pm 2.17$ \\
\hline$t p=$ & $<0.001$ & $<0.001$ & $<0.001$ & $<0.001$ & $<0.001$ & $<0.001$ \\
\hline \multicolumn{7}{|l|}{ MSSS (mean) } \\
\hline Initial & $4.59 \pm 3.02$ & $4.63 \pm 3.30$ & $4.62 \pm 2.96$ & $4.42 \pm 2.90$ & $4.28 \pm 3.00$ & $4.57 \pm 2.96$ \\
\hline Final & $4.21 \pm 3.04$ & $4.19 \pm 3.02$ & $4.11 \pm 2.92$ & $3.93 \pm 2.87$ & $3.55 \pm 2.93$ & $4.16 \pm 2.95$ \\
\hline$t p=$ & 0.002 & 0.001 & $<0.001$ & 0.002 & 0.005 & 0.001 \\
\hline
\end{tabular}

No.: number; EDSS: Expanded Disability Status Scale; MSSS: Multiple Sclerosis Severity Score; t: paired-sample Student's t-test.

the analysis comprised 158 alleles of HLA-DRB1, 158 of HLADPB1, 154 of HLA-DQB1, 108 of HLA-A, 116 of HLA-B and 156 of HLA-C (Table 1). Some patients were not tested for all alleles because of technical problems, and others yielded inconclusive results due to ambiguities.

Twelve different DMTs were used, and most patients were treated with more than one (Table 2). When more than one DMT was used by the same patient at a different time, each treatment was considered a single case during the period of the single drug. To be included in a specific group of DMT, we chose patients using only one DMT for at least six months (Table 2). If two drugs were used at same time, the patient was excluded from both groups of DMTs, except for corticosteroids during the relapses. If a corticosteroid was used concomitantly with another DMT during relapses, the patient was not included in the corticosteroid group but placed into the specific DMT being used. All patients in the corticosteroid group were not using any other DMT during the followup period. The following DMTs were used: corticosteroids, as soon as the signs or symptoms of relapses started in the first 24 hours (prednisone 80-140 mg, q.d. orally, followed by $1 \mathrm{mg}$ prednisone $/ \mathrm{kg} /$ day orally for 15 days and progressive withdrawal in one to two months or methylprednisolone $1.0 \mathrm{~g}$ IV for three or five days) without any DMT during the follow-up period; azathioprine $2-3 \mathrm{mg} / \mathrm{kg} /$ day orally and a progressive dosage increase until mean corpuscular volume was above
100 fL; interferon $\beta$-1a 22 mcg t.i.w., subcutaneous (subcut); interferon $\beta$-la 44 mcg t.i.w., subcut; interferon $\beta$-1a 30 mcg IM q.o.w.; interferon $\beta$-1b 0.25 mg q.o.d, subcut; glatiramer acetate $20 \mathrm{mg}$ q.d., subcut; mitoxantrone $120 \mathrm{mg}$ total dose in 18 months, IV; natalizumab 300 mg, q.m. IV; methotrexate 7.5-10 mg/week; fingolimod 0.5 mg, q.d., orally; or teriflunomide 14 mg, q.d., orally (Table 2).

The study was approved by the Ethics Committee for Research with Humans at the Hospital de Clínicas, Federal University of Paraná (CAAE: 0120.0.208.000-06, CEP: 1279.127/2006-09). All patients agreed to participate and signed a voluntary consent form.

\section{RESULTS}

The mean patient age was $34.08 \pm 10.59$ years, median 34.00 (ranging from 12 to 63 years) at the beginning of the DMT, and the mean treatment time was $46.18 \pm 46.03$ months, median 30 (ranging from 6 to 276 months).

The mean EDSS score was $2.53 \pm 1.87$, median 2.0 (ranging from 0 to 7.5 ) at the beginning of the DMT and $2.93 \pm 2.22$, median 2.50 (ranging from 0 to 8.5) at the end, indicating progression of disability $(\mathrm{p}<0.001)$.

The MSSS reduced in the majority of the patients for all the HLA alleles tested in all kinds of treatment (Table 3). 
Table 2. HLA classes and number of patients on each type of disease modifying therapy.

\begin{tabular}{|c|c|c|c|c|c|c|}
\hline Type of treatment & HLA-DRB1* (N) & HLA-DPB1* (N) & HLA-DQB1* (N) & HLA-A* $(N)$ & $\mathrm{HLA}-\mathrm{B} *(\mathrm{~N})$ & $\mathrm{HLA}-\mathrm{C} *(\mathrm{~N})$ \\
\hline Corticosteroids & 70 & 74 & 70 & 44 & 54 & 74 \\
\hline Duration & $60.43(7-276)$ & $56.27(7-276)$ & $57.66(7-276)$ & $64.32(12-276)$ & $65.07(12-276)$ & $57.46(7-276)$ \\
\hline MSSS Initial & $4.62(0.17-9.09)$ & $4.37(0.17-9.09)$ & $4.58(0.35-9.09)$ & $4.14(0.35-9.09)$ & $4.21(0.35-9.09)$ & $4.37(0.17-9.09)$ \\
\hline MSSS Final & $3.55(0.04-9.52)$ & $3.59(0.04-9.52)$ & $3.59(0.04-9.52)$ & $2.83(0.04-8.34)$ & $3.33(0.04-9.55)$ & $3.63(0.04-9.52)$ \\
\hline$t p=$ & 0.001 & 0.009 & 0.002 & 0.002 & 0.017 & 0.011 \\
\hline Azathioprine & 44 & 44 & 48 & 30 & 38 & 48 \\
\hline Duration & $66.86(6-216)$ & $66.36(6-216)$ & $63.88(6-216)$ & $73.80(6-216)$ & $67.47(6-216)$ & $69.13(6-216)$ \\
\hline MSSS Initial & $3.97(0.35-9.93)$ & $4.32(0.35-9.93)$ & $4.26(0.35-9.93)$ & $4.05(0.35-9.93)$ & $4.07(0.35-9.35)$ & $3.95(0.35-9.93)$ \\
\hline MSSS Final & $3.85(0.30-9.65)$ & $3.73(0.10-9.65)$ & $3.74(0.30-9.65)$ & $3.62(0.35-9.65)$ & $3.47(0.30-8.54)$ & $3.55(0.10-9.65)$ \\
\hline$t p=$ & 0.570 & 0.010 & 0.016 & 0.162 & 0.023 & 0.076 \\
\hline Interferon $\beta$-1a 22 mcg & 52 & 52 & 52 & 34 & 38 & 52 \\
\hline Duration & $33.85(6-102)$ & $42.27(6-147)$ & $43.85(6-147)$ & $35.65(6-101)$ & $38.58(6-102)$ & $43.85(6-147)$ \\
\hline MSSS Initial & $4.44(0.32-8.64)$ & $4.57(0.32-8.64)$ & $4.48(0.32-8.64)$ & $4.66(0.32-8.64)$ & $3.56(0.32-8.58)$ & $4.48(0.32-8.64)$ \\
\hline MSSS Final & $4.74(0.26-9.59)$ & $4.63(0.26-9.59)$ & $4.39(0.26-9.08)$ & $4.88(0.26-9.59)$ & $4.13(0.26-9.08)$ & $4.39(0.26-9.08)$ \\
\hline$t p=$ & 0.433 & 0.896 & 0.828 & 0.646 & 0.252 & 0.828 \\
\hline Interferon $\beta$-1a 44 mcg & 28 & 24 & 24 & 14 & 12 & 26 \\
\hline Duration & $35.57(9-96)$ & $35.58(9-96)$ & $37.67(9-96)$ & $34.71(18-96)$ & $36.50(9-96)$ & $35.08(9-96)$ \\
\hline MSSS Initial & $4.04(0.26-7.98)$ & $3.57(0.26-7.98)$ & $4.53(0.26-7.98)$ & $4.76(0.26-7.98)$ & $3.75(0.26-7.98)$ & $4.20(0.26-7.98)$ \\
\hline MSSS Final & $3.67(0.17-8.24)$ & $2.96(0.17-7.32)$ & $4.24(0.17-8.24)$ & $4.27(0.17-8.24)$ & $3.64(0.17-7.32)$ & $3.86(0.17-8.24)$ \\
\hline$t p=$ & 0.182 & 0.033 & 0.371 & 0.289 & 0.646 & 0.262 \\
\hline Interferon $\beta$-1a 30 mcg & 32 & 30 & 36 & 20 & 18 & 34 \\
\hline Duration & $39.13(6-90)$ & $31.87(8-80)$ & $32.61(6-90)$ & $35.10(12-80)$ & $35.67(12-80)$ & $36.24(6-90)$ \\
\hline MSSS Initial & $3.28(0.30-7.32)$ & $4.01(0.30-7.32)$ & $3.83(0.30-7.32)$ & $3.63(1.28-7.32)$ & $3.09(0.30-7.32)$ & $3.44(0.30-7.32)$ \\
\hline MSSS Final & $2.96(0.21-7.75)$ & $3.42(0.21-7.75)$ & $3.05(0.21-7.75)$ & $3.57(0.21-7.65)$ & $2.78(0.21-6.24)$ & $3.02(0.21-7.75)$ \\
\hline$t p=$ & 0.443 & 0.245 & 0.079 & 0.917 & 0.436 & 0.328 \\
\hline Interferon $\beta$-1b & 42 & 46 & 38 & 32 & 32 & 40 \\
\hline Duration & $40.43(7-132)$ & $54.13(7-132)$ & $52.74(7-132)$ & $43.94(7-132)$ & $53.94(7-132)$ & $51.20(7-132$ \\
\hline MSSS Initial & $4.69(0.30-9.63)$ & $4.50(0.30-9.63)$ & $4.58(0.30-9.63)$ & $4.45(0.30-9.63)$ & $4.32(0.30-9.63)$ & $4.55(0.35-9.63)$ \\
\hline MSSS Final & $4.44(0.25-8.83)$ & $4.21(0.25-8.83)$ & $4.22(0.25-8.83)$ & $3.81(0.25-8.83)$ & $3.76(0.25-8.83)$ & $4.35(0.32-8.83)$ \\
\hline$t p=$ & 0.468 & 0.370 & 0.346 & 0.111 & 0.167 & 0.575 \\
\hline Glatiramer Acetate & 42 & 36 & 38 & 32 & 28 & 36 \\
\hline Duration & $36.24(12-114)$ & $36.22(12-114)$ & $33.26(12-114)$ & $31.06(12-72)$ & $38.57(12-114)$ & $32.94(12-114)$ \\
\hline MSSS Initial & $4.72(0.25-9.59)$ & $4.94(0.25-9.59)$ & $4.46(0.25-8.70)$ & $4.52(0.25-9.59)$ & $4.51(0.25-8.70)$ & $4.95(0.45-8.70)$ \\
\hline MSSS Final & $3.97(0.17-8.50)$ & $4.39(0.17-8.50)$ & $4.03(0.17-8.38)$ & $4.10(0.17-8.50)$ & $3.52(0.17-8.38)$ & $4.38(0.21-8.38)$ \\
\hline$t p=$ & 0.019 & 0.189 & 0.277 & 0.094 & 0.036 & 0.172 \\
\hline Mitoxantrone & 16 & 16 & 14 & 6 & 10 & 16 \\
\hline Duration & $19.50(6-30)$ & $19.50(6-30)$ & $18.43(6-30)$ & $21.33(12-30)$ & $20.80(12-30)$ & $19.50(6-30)$ \\
\hline MSSS Initial & $8.11(6.61-9.80)$ & $8.11(6.61-9.80)$ & $8.01(6.61-9.80)$ & $7.47(6.61-8.83)$ & $7.81(6.61-9.80)$ & $8.11(6.61-9.80)$ \\
\hline MSSS Final & $8.27(6.14-9.95)$ & $8.27(6.14-9.95)$ & $8.17(6.14-9.95)$ & $7.14(6.14-8 ; 31)$ & $8.11(6.14-9.95)$ & $8.27(6.14-9.95)$ \\
\hline$t p=$ & 0.610 & 0.610 & 0.638 & 0.595 & 0.538 & 0.610 \\
\hline Natalizumab & 6 & 4 & 6 & 4 & 4 & 6 \\
\hline Duration & $21.33(7-29)$ & $18.00(7-29)$ & $21.33(7-29)$ & $18.00(7-29)$ & $18.00(7$ & $21.33(7-29)$ \\
\hline MSSS Initial & $6.92(5.79-8.38)$ & 7.08 (5.79-8.38) & $6.92(5.79-8.38)$ & $7.08(5.79-8.38)$ & 7.08 (5.79-8.38) & $6.92(5.79-8.38)$ \\
\hline MSSS Final & $4.90(2.10-7.33)$ & $4.71(2.10-7.33)$ & $4.90(2.10-7.33)$ & $4.71(2.10-7.33)$ & $4.71(2.10-7.33)$ & $4.90(2.10-7.33)$ \\
\hline$T p=$ & 0.026 & 0.063 & 0.026 & 0.063 & 0.063 & 0.026 \\
\hline Methotrexate & 4 & 4 & 4 & 2 & 4 & 4 \\
\hline Duration & $18.50(16-21)$ & $18.50(16-21)$ & $18.50(16-21)$ & $16.00(16-$ & $18.50(16$ & $18.50(16-$ \\
\hline MSSS Initial & $7.77(6.00-9.55)$ & $7.77(6.00-9.55)$ & $7.77(6.00-9.55)$ & $6.00(6.00-6.00)$ & $7.77(6-9.55)$ & $7.77(6.00-9.55)$ \\
\hline MSSS Final & $9.00(8.92-9.08)$ & $9.00(8.92-9.08)$ & $9.00(8.92-9.08)$ & $9.08(9.08-9.08)$ & 9.00 (8.92-9.08) & $9.00(8.92-9.08)$ \\
\hline$t p=$ & 0.458 & 0.458 & 0.458 & 0.157 & 0.458 & 458 \\
\hline Fingolimod & 0 & 0 & 2 & 2 & 0 & 0 \\
\hline Duration & - & - & $20.00(20-20)$ & $20.00(20-$ & - & - \\
\hline MSSS Initial & - & - & $1.04(1.04-1.04)$ & $1.04(1.04-1.04)$ & - & - \\
\hline MSSS Final & - & - & $0.21(0.21-0.21)$ & $0.21(0.21-0.21)$ & - & - \\
\hline$t p=$ & - & - & 0.157 & 0.157 & - & - \\
\hline Teriflunomide & 2 & 2 & 2 & 2 & 0 & 0 \\
\hline Duration & $6.00(6-6)$ & $6.00(6-6)$ & $6.00(6-6)$ & $6.00(6-6)$ & - & - \\
\hline MSSS Initial & $3.17(3.17-3.17)$ & $3.17(3.17-3.17)$ & $3.17(3.17-3.17)$ & $3.17(3.17-3.17)$ & - & - \\
\hline MSSS Final & $4.96(4.96-4.96)$ & $4.96(4.96-4.96)$ & $4.96(4.96-4.96)$ & $4.96(4.96-4.96)$ & - & - \\
\hline$t p=$ & 0.157 & 0.157 & 0.157 & 0.157 & - & - \\
\hline
\end{tabular}

N: number of patients; Duration: mean treatment duration (Months); MSSS: Multiple Sclerosis Severity Score; Mean (minimum - maximum). -: The correlation and $t$ could not be computed because the standard error of the difference is $0 ; t$ : paired-sample Student's $t$-test. $T$ : Wilcoxon signed-ranks test. 
The mean MSSS was $4.51 \pm 3.01$, median 4.30 (ranging from 0.17 to 9.93) at the beginning of the DMT and $4.06 \pm$ 3.01 (ranging from 0.04 to 9.95 ) at the end. A reduction in the MSSS was observed when all the patients were analyzed together, suggesting a stabilization or improvement of the disability ( $p<0.001)$. Most of the patients had a decrease in the MSSS despite the type of HLA or DMT, showing a beneficial effect of the therapy, but only few reached a statistically significant level (Table 3).

Some patients (two alleles) were treated with more than one DMT (total 1,794 occurrences comparing the MSSS between the initial and final score). The reduction of the MSSS occurred with most of the DMTs and specific alleles, such as HLA-DRB1 in 210/337, HLA-DPB1 in 204/331, HLA-DQB1 214/333, HLA-A 142/221, HLA-B 150/237 and HLA-C 204/335 (Table 3). However, the statistical relationships between the MSSS, HLA allele (subtypes of DRB1, DPB1, DQB1, A, B and C) and the DMT were significant $(p<0.05$ ) for only $15 / 245$ specific alleles with reduction of the MSSS (Table 4).

We found a statistically significant reduction in the MSSS, suggesting improvement of the disability for the following alleles: HLA-DRB1*15:01 (7/7), DPB1*04:01 (13/16), DQB1*02:01 (5/5) and DQB1*03:01 (8/8) treated with

Table 3. Number of patients with MSSS reduction and the most frequent HLA types in all DMT groups.

\begin{tabular}{|c|c|c|c|c|c|c|c|c|c|c|c|c|c|c|c|c|c|c|c|c|c|c|}
\hline HLA Alleles & $T$ & $\mathrm{R}$ & $\mathrm{T}$ & $\mathrm{R}$ & $T$ & $\mathrm{R}$ & $\mathrm{T}$ & $\mathrm{R}$ & $\mathrm{T}$ & $\mathrm{R}$ & $T$ & $\mathrm{R}$ & $T$ & $\mathrm{R}$ & $T$ & $\mathrm{R}$ & $\mathrm{T}$ & $\mathrm{R}$ & $\mathrm{T}$ & $\mathrm{R}$ & $\mathrm{T}$ & $\mathrm{R}$ \\
\hline DRB1* & \multicolumn{2}{|c|}{ All } & \multicolumn{2}{|c|}{$15: 01$} & \multicolumn{2}{|c|}{ 07:01 } & \multicolumn{2}{|c|}{ 03:01 } & \multicolumn{2}{|c|}{$13: 02$} & \multicolumn{2}{|c|}{$11: 04$} & \multicolumn{2}{|c|}{ 04:04 } & \multicolumn{2}{|c|}{ 13:01 } & \multicolumn{2}{|c|}{$16: 01$} & \multicolumn{2}{|c|}{$14: 01$} & \multicolumn{2}{|c|}{ 11:01 } \\
\hline Number of patients & 337 & 210 & 40 & 29 & 32 & 19 & 32 & 19 & 22 & 7 & 17 & 10 & 14 & 12 & 13 & 9 & 13 & 10 & 11 & 8 & 11 & 9 \\
\hline DPB1* & \multicolumn{2}{|c|}{ All } & \multicolumn{2}{|c|}{ 04:01 } & \multicolumn{2}{|c|}{ 02:01 } & \multicolumn{2}{|c|}{ 04:02 } & \multicolumn{2}{|c|}{ 03:01 } & \multicolumn{2}{|c|}{ 10:01 } & \multicolumn{2}{|c|}{ 23:01 } & \multicolumn{2}{|c|}{ 01:01 } & \multicolumn{2}{|c|}{$14: 01$} & \multicolumn{2}{|c|}{ 05:01 } & \multicolumn{2}{|c|}{ 13:01 } \\
\hline Number & 331 & 204 & 79 & 50 & 32 & 20 & 31 & 16 & 27 & 14 & 24 & 12 & 16 & 12 & 19 & 11 & 10 & 5 & 10 & 8 & 8 & 3 \\
\hline DQB1* & \multicolumn{2}{|c|}{ All } & \multicolumn{2}{|c|}{$06: 02$} & \multicolumn{2}{|c|}{ 03:02 } & \multicolumn{2}{|c|}{ 03:01 } & \multicolumn{2}{|c|}{ 02:01 } & \multicolumn{2}{|c|}{ 04:02 } & \multicolumn{2}{|c|}{ 05:01 } & \multicolumn{2}{|c|}{ 05:03 } & \multicolumn{2}{|c|}{ 06:03 } & & & 06 & \\
\hline Number of patients & 333 & 214 & 44 & 31 & 47 & 26 & 34 & 24 & 30 & 21 & 25 & 14 & 24 & 11 & 15 & 10 & 13 & 7 & 12 & 6 & 12 & 10 \\
\hline HLA-A* & A & II & & 02 & & & 02 & 01 & 01 & 01 & 23 & 01 & 11 & & 68 & & & & & & 25 & \\
\hline Number of patients & 221 & 142 & 31 & 21 & 30 & 17 & 28 & 16 & 24 & 14 & 12 & 8 & 9 & 4 & 9 & 7 & 8 & 6 & 7 & 6 & 6 & 4 \\
\hline HLA-B* & A & II & & & & & 07 & 02 & 14 & 02 & & 02 & 49 & & & & & & & & 40 & \\
\hline Number of patients & 237 & 150 & 28 & 20 & 23 & 15 & 19 & 12 & 12 & 5 & 9 & 4 & 7 & 3 & 7 & 3 & 6 & 5 & 7 & 5 & 6 & 3 \\
\hline HLA-C* & $A$ & II & & & & & 07: & 01 & 08 & 02 & 05 & 01 & 06 & 02 & 03 & & & & & & 15 & \\
\hline Number of patients & 335 & 204 & 55 & 33 & 41 & 26 & 24 & 13 & 26 & 15 & 24 & 15 & 20 & 11 & 16 & 9 & 11 & 7 & 12 & 5 & 11 & 9 \\
\hline
\end{tabular}

DMT: Disease Modifying Therapy; MSSS: Multiple Sclerosis Severity Score; T: total number of patients treated with DMT; R: number of patients with a reduction in MSSS.

Table 4. HLA alleles with a statistically-significant relationship with the DMT.

\begin{tabular}{|c|c|c|c|c|c|c|}
\hline DMT & $\begin{array}{c}\text { Number } \\
\text { patients*/Total }\end{array}$ & Age (years) & $\begin{array}{l}\text { Duration treatment** } \\
\text { (months) }\end{array}$ & MSSS initial*** & MSSS final $* * \star$ & $\mathrm{p}=T$ \\
\hline \multicolumn{7}{|l|}{ HLA alleles* } \\
\hline \multicolumn{7}{|l|}{ Corticosteroids } \\
\hline DRB1*15:01 & $7 / 74$ & $29.43 \pm 9.97$ & $96.29(12-278)$ & $5.99 \pm 2.62$ & $2.74 \pm 1.85$ & 0.018 \\
\hline DPB1*04:01 & $16 / 74$ & $32.75 \pm 9.48$ & $90.81(12-276)$ & $4.55 \pm 3.20$ & $2.73 \pm 2.84$ & 0.023 \\
\hline $\mathrm{DQB} 1 * 02: 01$ & $5 / 70$ & $34.40 \pm 4.27$ & $78.20(20-218)$ & $4.87 \pm 1.66$ & $2.79 \pm 1.24$ & 0.043 \\
\hline $\mathrm{DQB} 1 * 03: 01$ & $8 / 70$ & $35.13 \pm 12.03$ & $82.25(17-128)$ & $5.00 \pm 3.76$ & $1.96 \pm 1.54$ & 0.011 \\
\hline \multicolumn{7}{|l|}{ Azathioprine } \\
\hline DRB1*03:01 & $5 / 44$ & $43.40 \pm 8.23$ & $70.00(6-144)$ & $7.73 \pm 3.02$ & $6.94 \pm 3.65$ & 0.043 \\
\hline DPB1*04:01 & $7 / 44$ & $40.71 \pm 5.34$ & $86.29(19-144)$ & $4.35 \pm 4.10$ & $2.68 \pm 2.30$ & 0.042 \\
\hline $\mathrm{DQB} 1 * 03: 02$ & $7 / 48$ & $33.00 \pm 14.90$ & $25.71(6-67)$ & $3.58 \pm 2.59$ & $2.64 \pm 2.43$ & 0.018 \\
\hline DQB1*06:02 & $7 / 48$ & $44.57 \pm 11.07$ & $94.71(18-144)$ & $6.83 \pm 3.45$ & $5.40 \pm 3.82$ & 0.018 \\
\hline$C * 07: 02$ & $5 / 48$ & $38.60 \pm 6.76$ & $93.40(21-144)$ & $5.74 \pm 4.23$ & $3.88 \pm 3.14$ & 0.043 \\
\hline \multicolumn{7}{|c|}{ Interferon $\beta$-1a $22 \mu g$} \\
\hline DRB1*11:04 & $6 / 52$ & $25.17 \pm 11.42$ & $48.33(20-80)$ & $6.80 \pm 1.83$ & $5.24 \pm 3.11$ & 0.027 \\
\hline DQB1*03:01 & $5 / 52$ & $28.80 \pm 10.96$ & $50.60(20-80)$ & $6.10 \pm 2.18$ & $4.82 \pm 2.82$ & 0.043 \\
\hline $\mathrm{DQB} 1 * 03: 02$ & $5 / 52$ & $30.00 \pm 8.74$ & $29.60(12-50)$ & $4.01 \pm 3.17$ & $3.42 \pm 3.17$ & 0.042 \\
\hline \multicolumn{7}{|c|}{ Interferon $\beta$-1a $30 \mu g$} \\
\hline DPB1*02:01 & $5 / 30$ & $42.20 \pm 18.78$ & $33.80(22-49)$ & $3.29 \pm 1.00$ & $2.03 \pm 1.11$ & 0.042 \\
\hline$C * 05: 01$ & $5 / 34$ & $35.40 \pm 6.30$ & $40.40(6-90)$ & $2.18 \pm 1.37$ & $1.36 \pm 1.36$ & 0.042 \\
\hline \multicolumn{7}{|l|}{ Interferon $\beta$-1b } \\
\hline $\mathrm{DQB1} 1 * 02: 01$ & $5 / 38$ & $31.50 \pm 10.93$ & $63.67(18-118)$ & $6.25 \pm 3.55$ & $4.47 \pm 3.13$ & 0.046 \\
\hline
\end{tabular}

DMT: disease modifying therapies; *: Patients with statistical significance; MSSS: Multiple Sclerosis Severity Score; Initial: score at the beginning of treatment; Final: score at the end of treatment; **: Mean (maximum-minimum); $* \star *:$ Mean \pm standard deviation; T: Wilcoxon signed-ranks test. 
corticosteroids; HLA-DRB1*03:01 (5/5); HLA-DPB1*04:01 (6/7), DQB1*03:02 (7/7) and DQB1*06:02 (7/7), HLAC*07:02 (5/5) treated with azathioprine; HLA-DRB1*11:04 (6/6), DQB1*03:01 (5/5) and DQB1*03:02 (5/5) treated with interferon $\beta$-1a 22 mcg; HLA-DPB1*02:01 (5/5); HLA$C^{*} 05: 01(5 / 5)$ treated with interferon $\beta$-1a 30 mcg; HLADQB1*02:01 (5/6) treated with interferon $\beta$-1b (Table 4). For the other alleles, there was no statistically significant relationship (Table 4).

\section{DISCUSSION}

High-resolution HLA sequencing techniques have been described for some years and have enabled many different HLA alleles to be identified in various populations ${ }^{21,22}$. Because of its multi-ethnic nature, the southern Brazilian population, which was previously classified as white skinned (95.6\%) and black skinned from Afro-descendence (3.2\%), has a great diversity of HLA alleles as well as HLA profiles different from those of the European populations. In our study, the HLA profile revealed that diversity, for example, the HLADRB1*15:01 found more frequently in Europeans, had a frequency of only $14 \%$ in MS patients and in $8.1 \%$ in controls in south Brazil ${ }^{10}$. Many different alleles were also found in the present study, resulting in fewer patients for each type of treatment (Table 2).

Regardless of the DMT used, the EDSS increased in all the groups in our study when these were analyzed together, and showed a statistically significant relationship with disease progression and time of onset, in agreement with the current literature ${ }^{1,3}$. The EDSS is a useful and widely-used scale to measure the MS progression ${ }^{17}$. Invariably, most patients increased in their disability despite the type of therapy; this being more intense in the first years of the disease due to inflammatory phase activity before their entry into the degenerative phase ${ }^{3,23}$. Most of the studies on DMTs assessing the disability using the EDSS were over a two-year study period. These studies showed variable benefits comparing placebo with other DMTs or corticosteroids, interferon $\beta$-1a 44 mcg t.i.d., interferon $\beta$-1a 30 mcg IM weekly, mitoxantrone, natalizumab, fingolimod and teriflunomide ${ }^{24}$. There has been insufficient evidence for azathioprine, interferon $\beta$-1b 8 MIUs and methotrexate. No benefit was found with the use of glatiramer acetate ${ }^{24}$.

We chose to use the MSSS because it relates the EDSS to the distribution of disabilities in patients with comparable disease durations ${ }^{18}$. Analysis of the MSSS for our patients revealed a reduction in disability when all the HLA alleles and the DMTs were analyzed as one group (all patients together). A large proportion of our patients showed a reduction in this score, and this varied according to the allele type in each HLA class. For some specific HLA types, there was a reduction in MSSS when our patients were treated with corticosteroids, azathioprine, interferon $\beta$-1a 22 mcg, interferon $\beta$-1a $30 \mathrm{mcg}$ and interferon $\beta$-1b (Table 4).

Our data found a relationship between the use of a corticosteroid alone over 12 to 278 months, and a decrease on the MSSS in patients with HLA-DRB1*15:01, DPB1*04:01, DQB1*02:01 and DQB1*03:01, suggesting stabilization or improvement of the disability. In relapses, IV corticosteroid therapy has led to a high rate of complete or partial recovery of symptoms in short-term follow-up ${ }^{25}$. However, some patients with specific HLA alleles have shown good response with reduction or slow progression of the disability after several years using only prednisone in multiple "short time" therapies (2-3 weeks), instead of a high dose for a few days ${ }^{26}$. Nevertheless, our review of the literature failed to reveal any study correlating the HLA and prednisone therapy.

The patients with HLA-DRB1*03:01, HLA-DPB1*14:01, DQB1*06:02 and DQB1*03:02 treated with azathioprine showed a reduction on the MSSS ( $p=0.018$ to 0.043 ) indicating improvement or stabilization of the disability. Despite that azathioprine is not widely used in MS treatment, some studies have shown similar effects of the immunomodulatory drugs for relapses, progression of disability and number of lesions on $\mathrm{MRI}^{27-30}$. A study using azathioprine and serological HLA typing showed progression of the disease during treatment in patients with HLA-A1-B8, HLA-B8-DR3 and HLA-A1-B8-DR3, and no difference for HLA-B7 and HLA-DR2 ${ }^{31}$. Later, a trial with azathioprine, which included some data on serological HLA typing, revealed a small benefit, as measured by the EDSS, for patients with HLA-DR3 and HLA-DR2 after a three-year period but without a relationship with the HLA subtype $^{28}$. However, all these studies used low resolution techniques to identify the HLA type.

The patients treated with interferon $\beta$-1a $22 \mu \mathrm{g}$ (HLA$\mathrm{DRB}^{*}$ 11:04, DQB1*03:01 and DQB1*03:02), interferon $\beta$-1a 30 $\mu \mathrm{g}$ (HLA-DPB1*02:01 and HLA-C*05:01) and interferon $\beta$-1b (DQB1*02:01) decreased their MSSS ( $\mathrm{p}<0.043$ to $\mathrm{p}<0.027$ ), suggesting improvement or stabilization of their disability (duration of treatment six to 90 months). In an earlier study that used low-resolution HLA typing techniques, a reduction in MS relapses after one year of treatment with interferon $\beta$-1a (22 mcg t.i.w. subcut) was found in patients with HLA-DRB $1 * 03$, HLA-DQB1*03 and HLA-DQB1*02 ${ }^{32}$. In another study, a beneficial response (reduced disease relapses and stabilization of EDSS scores in the two years of follow-up) was reported with interferon $\beta$-la (20 $\mu$ g t.i.w., IM) in patients with HLA-DRB1*04 or the HLA-A*03-DRB1*04 haplotype ${ }^{13}$.

We did not find any statistical relationship in our patients using glatiramer acetate. However, there is a report that glatiramer acetate and the presence of HLA-DR15 and HLA-DQ6, or absence of HLA-DR17 and HLA-DQ2, prevents relapses and halts the disease progression ${ }^{33}$. Also, the presence of HLA-DRB1*15:01, HLA-DQB2/DOB and HLA-DOB/TAP2, as well as several single nucleotide polymorphisms (SNPs) in 
genes involved in the inflammatory cascade, were associated with a reduction in the annual relapse rate within two years ${ }^{14}$.

Methotrexate is not widely prescribed for MS at the present time, but although previous studies have shown some efficacy on specific clinical grounds, in spite of its efficacy being inferior to interferon $\beta$-1a, methotrexate is used more frequently as add-on therapy ${ }^{34}$. No study was found correlating methotrexate and HLAs in MS patients.

A genome-wide pharmacogenetics investigation found statistically significant differences in response to interferon $\beta$ therapy between some individuals with different SNPs in a population from France and Spain with a predominant HLA-DRB1*15:01 genotype $(38 \%)^{35}$. Modest relationships were observed between SNPs from several genes outside the HLA system and response to DMTs in European and American populations. The relationship was found in MS patients who were responders and non-responders to interferons and glatiramer acetate, in some patients with HLA-DRB1*15:01, DQB2 and DOB/TAP2 ${ }^{7,33}$. However, the one limitation of these studies was because they did not compare the classic HLA types versus SNPs in the other genes $^{14,36}$. These SNPs were different in each study, suggesting that there are factors other than the genetic component mediating the response to immunomodulators ${ }^{8,35,37,38}$.

In our study, the fact that there were many different HLA types "diluted" the number of patients for each allele, making it difficult to carry out a statistical analysis in our population who had allele distributions different from Europeans ${ }^{10}$. Ours is a report of a real-world outpatient clinic, with the inclusion of patients with six months of treatment, showing that most of the DMTs had an effect on the disability in the long term, in the majority of patients, even without statistical significance (Table 4). This was not an artificial controlled trial study, where the patients who did not fit the protocol were excluded. The patients with six months of treatment changed to a different DMT because they had collateral effects, allergy, severe depression, more than one severe exacerbation, increased their EDSS by several points or developed new lesions on magnetic resonance imaging.

We failed to find similar studies in the literature that compared HLA subtypes using high-resolution HLA typing techniques and correlated the HLA type with the disability score, disease duration and different forms of therapy. In addition, the HLA profiles in the populations of previous studies were different from those of our population and the interplay of the innumerable genes outside the MHC but present in several sub-sets of cells involved in the pathogenesis of MS, may interfere with the results ${ }^{39,40}$. Also, there is growing evidence of external factors-such as UV radiation, vitamin $\mathrm{D}$, gut microbiomes, viral infections, smoke, sodium intake and others-may have some influence on the pathogenesis of MS and may be more important than the immune genetic profile of the patient in the efficacy of DMTs ${ }^{41-44}$.

In conclusion, our study showed a relationship between the HLA and the effect of DMTs on some HLA class I and II alleles in some patients. We observed a decrease in the MSSS for certain HLA genotypes, which might reflect a better response to different DMTs in a few patients. These results should be interpreted with caution because of the small number of patients with some types of HLA and DMTs.

\section{ACKNOWLEDGMENTS}

Raquel C. Arndt, B.Sc., for her technical assistance in the HLA analysis (Neurology Service, Hospital de Clínicas, UFPR, Curitiba, Brazil).

\section{References}

1. Cross AH, Naismith RT. Established and novel diseasemodifying treatments in multiple sclerosis. J Intern Med. 2014 Apr;275(4):350-63. https://doi.org/10.1111/joim.12203

2. Wingerchuk DM, Carter JL. Multiple sclerosis: current and emerging disease-modifying therapies and treatment strategies. Mayo Clin Proc. 2014 Feb;89(2):225-40. https://doi.org/10.1016/j.mayocp.2013.11.002

3. Werneck LC, Lorenzoni PJ, Radünz VA, Utiumi MA, Kay CS, Scola RH. Influence of treatment in multiple sclerosis disability: an open, retrospective, nonrandomized long-term analysis. Arq Neuropsiquiatr. 2010 Aug;68(4):511-21. https://doi.org/10.1590/S0004-282X2010000400008

4. Comabella M, Fernández-Arquero M, RíoJ, Guinea A, Fernández M, Cenit MC et al. HLA class I and II alleles and response to treatment with interferon-beta in relapsing-remitting multiple sclerosis. J Neuroimmunol. 2009 May;210(1-2):116-9. https://doi.org/10.1016/j.jneuroim.2009.01.012

5. Grossman I, Avidan N, Singer C, Goldstaub D, Hayardeny L, Eyal E et al. Pharmacogenetics of glatiramer acetate therapy for multiple sclerosis reveals drug-response markers. Pharmacogenet Genomics. 2007 Aug;17(8):657-66. https://doi.org/10.1097/FPC.0b013e3281299169
6. Fernández O, Fernandéz V, Mayorga C, Guerrero M, Leon A, Tamayo JA. HLA class II and response to inferferon-beta in multiple sclerosis. Acta Neurol Scand 2005;112:391-4. https://doi.org/10.1111/j.1600-0404.2005.00415.x

7. Samadzadeh S, Tabibian E, Sabokbar T, Shakoori A, Dehgolan SR, Armaki SA, et al. HLA-DRB1 does not have a role in clinical response to interferon-beta among Iranian multiple sclerosis patients. J Neurol Sci. 2015 May;352(1-2):37-40. https://doi.org/10.1016/j.jns.2015.03.004

8. Søndergaard HB, Petersen ER, Magyari M, Sellebjerg F, Oturai AB. Genetic burden of MS risk variants distinguish patients from healthy individuals but are not associated with disease activity. Mult Scler Relat Disord. 2017 Apr;13:25-7. https://doi.org/10.1016/j.msard.2017.01.015

9. Murphy K, Travers P, Walport M. Janeways's Immunobiology. 7th ed. New York: Garland Sciences; 2008.

10. Werneck LC, Lorenzoni PJ, Arndt RC, Kay CS, Scola RH. The immunogenetics of multiple sclerosis. The frequency of HLAalleles class 1 and 2 is lower in Southern Brazil than in the European population. Arq Neuropsiquiatr. 2016 Aug;74(8):607-16. https://doi.org/10.1590/0004-282X20160100 
11. Buck D, Cepok S, Hoffmann S, Grummel V, Jochim A, Berthele A et al. Influence of the HLA-DRB1 genotype on antibody development to interferon beta in multiple sclerosis. Arch Neurol. 2011 Apr;68(4):480-7. https://doi.org/10.1001/archneurol.2011.65

12. Barbosa MD, Vielmetter J, Chu S, Smith DD, Jacinto J. Clinical link between MHC class II haplotype and interferon-beta (IFN-beta) immunogenicity. Clin Immunol. 2006 Jan;118(1):42-50. https://doi. org/10.1016/j.clim.2005.08.017 PMID:16260183

13. Mazdeh M, Taheri M, Sayad A, Bahram S, Omrani MD, Movafagh A et al. HLA genes as modifiers of response to IFN- $\beta$-1a therapy in relapsing-remitting multiple sclerosis. Pharmacogenomics. 2016 Apr;17(5):489-98. https://doi.org/10.2217/pgs.16.2

14. Ross CJ, Towfic F, Shankar J, Laifenfeld D, Thoma M, Davis M et al. A pharmacogenetic signature of high response to Copaxone in latephase clinical-trial cohorts of multiple sclerosis. Genome Med. 2017 May;9(1):50. https://doi.org/10.1186/s13073-017-0436-y

15. Polman $\mathrm{CH}$, Reingold SC, Banwell B, Clanet M, Cohen JA, Filippi M et al. Diagnostic criteria for multiple sclerosis: 2010 revisions to the McDonald criteria. Ann Neurol. 2011 Feb;69(2):292-302. https://doi. org/10.1002/ana.22366

16. Lublin FD, Reingold SC, Cohen JA, Cutter GR, Sørensen PS, Thompson AJ et al. Defining the clinical course of multiple sclerosis: the 2013 revisions. Neurology. 2014 Jul;83(3):278-86. https://doi.org/10.1212/ WNL.0000000000000560

17. Kurtzke JF. Rating neurologic impairment in multiple sclerosis: an expanded disability status scale (EDSS). Neurology. 1983 Nov;33(11):1444-52. https://doi.org/10.1212/WNL.33.11.1444

18. Roxburgh RH, Seaman SR, Masterman T, HensiekAE, Sawcer SJ, Vukusic S et al. Multiple Sclerosis Severity Score: using disability and disease duration to rate disease severity. Neurology. 2005 Apr;64(7):1144-51. https://doi.org/10.1212/01.WNL.0000156155.19270.F8

19. de Winter JC. Using t-test with extremely small sample size. Pract Assess Res Eval. 2013;18(10):1-12.

20. Iman A, Mohamammed U, Abanyam CM. On consistency and limitation of paired t-test: sign and Wilcoxon sign rank test. IOSR Journal of Mathematics. 2014;10:1-6. https://doi.org/10.9790/5728-10140106

21. HLA Informatics Group. The WHO Nomenclature Committee for Factors of the HLA System. London: HLA; Release 3.33.0. 2018 [cited 2018 Jul 11]. Available from: http://www.ebi.ac.uk/imgt/hla/

22. Robinson J, Halliwell JA, McWilliam H, Lopez R, Parham P, Marsh SG. The IMGT/HLA database. Nucleic Acids Res. 2013 Jan;41(Database issue):D1222-7. https://doi.org/10.1093/nar/gks949

23. Leray E, Yaouanq J, Le Page E, Coustans M, Laplaud D, Oger

$J$ et al. Evidence for a two-stage disability progression in multiple sclerosis. Brain. $2010 \mathrm{Jul}$;133(Pt 7):1900-13. https://doi.org/10.1093/brain/awq076

24. Rae-Grant A, Day GS, Marrie RA, Rabinstein A, Cree BA, Gronseth GS et al. Practice guideline recommendations summary: Diseasemodifying therapies for adults with multiple sclerosis: Report of the Guideline Development, Dissemination, and Implementation Subcommittee of the American Academy of Neurology [Supplemental Data.]. Neurology. 2018 Apr;90(17):777-88. https:// doi.org/10.1212/WNL.0000000000005347

25. Stoppe M, Busch M, Krizek L, Then Bergh F. Outcome of MS relapses in the era of disease-modifying therapy. BMC Neurol. 2017 Aug;17(1):151. https://doi.org/10.1186/s12883-017-0927-x

26. Johnson LN, Morey SS. Repeated intervals of high-dose corticosteroid: an overlooked therapy in multiple sclerosis. Mo Med. 2005 Jan-Feb;102(1):47-50.

27. Etemadifar M, Janghorbani M, Shaygannejad V. Comparison of interferon beta products and azathioprine in the treatment of relapsing-remitting multiple sclerosis. J Neurol. 2007 Dec;254(12):1723-8. https://doi.org/10.1007/s00415-007-0637-1

28. British and Dutch Multiple Sclerosis Azathioprine Trial Group. Double-masked trial of azathioprine in multiple sclerosis. Lancet. 1988 Jul;2(8604):179-83. https://doi.org/10.1016/S0140-6736(88)92286-6

29. Casetta I, Iuliano G, Filippini G. Azathioprine for multiple sclerosis. J Neurol Neurosurg Psychiatry. 2009 Feb;80(2):131-2. https://doi. org/10.1136/jnnp.2008.144972

30. Massacesi L, Parigi A, Barilaro A, Repice AM, Pellicanò G, Konze A et al. Efficacy of azathioprine on multiple sclerosis new brain lesions evaluated using magnetic resonance imaging. Arch Neurol. 2005 Dec;62(12):1843-7. https://doi.org/10.1001/archneur.62.12.1843

31. Madigand M, Oger JJ, Fauchet R, Sabouraud O, Genetet B. HLA profiles in multiple sclerosis suggest two forms of disease and the existence of protective haplotypes. J Neurol Sci. 1982 Mar;53(3):51929. https://doi.org/10.1016/0022-510X(82)90248-9

32. Flechter S, Klein T, Pollak L. Influence of histocompatibility genes on disease susceptibility and treatment response in patients with relapsing-remitting multiple sclerosis treated with interferon $\beta-1 \mathrm{a}$. Neurol Int. 2011 Jun;3(1):e5. https://doi.org/10.4081/ni.2011.e5

33. Dhib-Jalbut S, Valenzuela RM, Ito K, Kaufman M, Ann Picone M, Buyske S. HLA DR and DQ alleles and haplotypes associated with clinical response to glatiramer acetate in multiple sclerosis. Mult Scler Relat Disord. 2013 Oct;2(4):340-8. https://doi.org/10.1016/j. msard.2013.02.005

34. Ashtari F, Savoj MR. Effects of low dose methotrexate on relapsingremitting multiple sclerosis in comparison to Interferon $\beta-1 \alpha$ : a randomized controlled trial.J Res Med Sci. 2011 Apr;16(4):457-62.

35. Byun E, Caillier SJ, Montalban X, Villoslada P, Fernández O, Brassat $D$ et al. Genome-wide pharmacogenomic analysis of the response to interferon beta therapy in multiple sclerosis. Arch Neurol. 2008 Mar;65(3):337-44. https://doi.org/10.1001/archneurol.2008.47

36. Mahurkar S, Moldovan M, Suppiah V, Sorosina M, Clarelli F, Liberatore $G$ et al. Response to interferon-beta treatment in multiple sclerosis patients: a genome-wide association study. Pharmacogenomics J. 2017 Jul;17(4):312-8. https://doi.org/10.1038/ tpj.2016.20

37. Comabella M, Craig DW, Morcillo-Suárez C, Río J, Navarro A, Fernández M et al. Genome-wide scan of 500,000 single-nucleotide polymorphisms among responders and nonresponders to interferon beta therapy in multiple sclerosis. Arch Neurol. 2009 Aug;66(8):9728. https://doi.org/10.1001/archneurol.2009.150

38. Kulakova OG, Tsareva EY, Lvovs D, Favorov AV, Boyko AN, Favorova OO. Comparative pharmacogenetics of multiple sclerosis: IFN- $\beta$ versus glatiramer acetate. Pharmacogenomics. 2014 Apr;15(5):679-85. https://doi.org/10.2217/pgs.14.26

39. Kieseier BC. The mechanism of action of interferon- $\beta$ in relapsing multiple sclerosis. CNS Drugs. 2011 Jun;25(6):491-502. https://doi. org/10.2165/11591110-000000000-00000

40. Sawcer S, Hellenthal G, Pirinen M, Spencer CC, Patsopoulos NA, Moutsianas L et al. Genetic risk and a primary role for cellmediated immune mechanisms in multiple sclerosis. Nature. 2011 Aug;476(7359):214-9. https://doi.org/10.1038/nature10251

41. Pierrot-Deseilligny C, Souberbielle JC. Contribution of vitamin $D$ insufficiency to the pathogenesis of multiple sclerosis. Ther Adv Neurol Disorder. 2013;6(2):81-116. https://doi. org/10.1177/1756285612473513

42. Wergeland S, Myhr KM, Løken-Amsrud KI, Beiske AG, Bjerve KS, Hovdal $\mathrm{H}$ et al. Vitamin D, HLA-DRB1 and Epstein-Barr virus antibody levels in a prospective cohort of multiple sclerosis patients. Eur J Neurol. 2016 Jun;23(6):1064-70. https://doi.org/10.1111/ene.12986

43. Jangi S, Gandhi R, Cox LM, Li N, Glehn F, Yan R et al. Alterations of the human gut microbiome in multiple sclerosis. Nat Commun. 2016 Jun;7:12015. https://doi.org/10.1038/ncomms12015

44. Berg-Hansen P, Moen SM, Sandvik L, Harbo HF, Bakken IJ, Stoltenberg $\mathrm{C}$ et al. Prevalence of multiple sclerosis among immigrants in Norway. Mult Scler. 2015 May;21(6):695-702. https:// doi.org/10.1177/1352458514554055 